\title{
Comparison of routine cytology findings with conventional Ziehl-Neelsen method and fluorescent method for detection of acid-fast bacilli in fine needle aspirates from suspected cases of tuberculous lymphadenitis
}

\author{
Goswami A.P. ${ }^{1}$, Brahmbhatt S. ${ }^{2}$, Khandkar A. ${ }^{3}$, Zinzala U. ${ }^{4}$, Gondliya J. ${ }^{5}$, Goswami P. ${ }^{6}$ \\ ${ }^{1}$ Dr. Alpeshpuri P. Goswami, Associate Professor \& Laboratory Quality Manager, ${ }^{2}$ Dr. Smit Brahmbhatt, ${ }^{3}$ Dr. Abhijeet \\ Khandkar, ${ }^{4}$ Dr. Umesh Zinzala, ${ }^{5}$ Dr. Jignesh Gondliya, ${ }^{6}$ Dr. Parth Goswami, Assistant Professor, Pathology Department, \\ Government Medical College, Bhavnagar; ${ }^{2,3,4,5}$ Postgraduate Resident Doctor, all the authors are affiliated with the Department \\ of Pathology, Government Medical College, Bhavnagar, Gujarat, India.
}

Corresponding Author: Dr. Smit Brahmbhatt, Room No.303, PG 3 Hostel, Sir T. General Hospital Campus, Bhavnagar. Gujarat, India. Email: smitbrahmbhatt14@gmail.com

\begin{abstract}
Objective: This study aimed to correlate the modified fluorescence stain method and conventional $\mathrm{ZN}$ method with routine cytology findings on lymph node aspirates for the detection of AFB in suspected cases of tuberculous lymphadenitis. Material and Methods: After adequate fine needle aspiration, aspirates were examined for cytology diagnosis, studied with ZiehlNeelsen and Auramine-rhodamine Fluorescent stain. Cytological findings, ZN stain observations and grading were compared with fluorescent stain grading. Result: In the present study, 36 cases were reported positive by fluorescent staining method for M. tuberculosis. Out of 43 granulomatous lymphadenitis cases diagnosed by FNAC, 29 cases were reported positive for acid fast bacilli by Z-N staining. 11 cases were found as false negative (22\%). Sensitivity and specificity of Z-N method was found to be $70.27 \%$ and $76.92 \%$ respectively. And for cytological method it was $96.77 \%$ and $31.57 \%$ respectively. Conclusion: There are problems in arriving at an absolute diagnosis in certain cases of tuberculous lymphadenitis when the aspirate shows polymorphous picture with occasional epithelioid cells and absence of typical Langhans giant cell or caseous necrosis. Microscopy has many advantages when it comes to speed $\&$ feasibility, its sensitivity could be improved by adjuvant methods.
\end{abstract}

Keywords: Tuberculosis, Lymphadenopathy, Cytology, Fluorescent, Zeihl-neelsen, Z-N staining, Acid fast bacilli.

\section{Introduction}

Tuberculosis continues to be a major health problem in developing countries. It is caused by bacteria of the Mycobacterium tuberculosis complex, is one of the oldest diseases known to affect humans and a major cause of death worldwide [1]. India has the highest burden of tuberculosis in the world as reflected by the World Health Organization statistics for 2011, giving an estimated incidence of 2.2 million cases of tuberculosis for India of a global incidence of 8.7 million cases [2]. M. tuberculosis is a rod-shaped, non-spore-forming, thin aerobic bacterium measuring $0.5 \mathrm{X}$ $3 \mu \mathrm{m}$.

Mycobacteria are acid-fast bacilli. Acid fastness is due mainly to the organisms' high content of mycolic acids, long-chain cross-linked fatty acids, and other cell-wall lipids [1]. Lymphadenopathy is the most common presentation of extra pulmonary TB. Lymph node TB

Manuscript received: $20^{\text {th }}$ November 2019

Reviewed: $30^{\text {th }}$ November 2019

Author Corrected: $6^{\text {th }}$ December 2019

Accepted for Publication: $12^{\text {th }}$ December 2019 presents as painless swelling of the lymph nodes, most commonly at posterior cervical and supraclavicular sites (a condition historically referred to as scrofula) [1]. Fineneedle aspiration cytology (FNAC) of lymph nodes in TB has varied cytomorphological features. Routinely hematoxylin \& eosin staining is done for each smear but conventional Ziehl-Neelsen staining is necessary to reveal the acid fastness of the tubercle bacilli. The conventional Z$\mathrm{N}$ staining method for acid-fast bacilli (AFB) plays a key role in the diagnosis and also for the monitoring of treatment in TB. But, its major disadvantage is low sensitivity ranging from $20 \%$ to $43 \%$ [3].

Mycobacterial culture is the reference method for the detection of tubercle bacilli but it is time consuming and requires specialized safety procedures in laboratories. Serological techniques have the disadvantage of lack of sensitivity and specificity. Auramine orange (AO) staining is a commonly used test on both cytological smears and histological sections for the detection of acid-fast bacilli. It

Pathology Update: Tropical Journal of Pathology \& Microbiology Available online at: www.medresearch.in 1015|P a g e 
has been widely reported as a sensitive stain with high positive predictive value in comparison to culture techniques. But there is little literature related to the comparison of Auramine-Rhodamine stained smears under fluorescent microscopy with Z-N stain for the detection of AFB in lymph node aspirates [4].

This study aimed to correlate the modified fluorescence stain method and conventional $\mathrm{ZN}$ method with routine cytology findings on lymph node aspirates for the detection of AFB in suspected cases of tuberculous lymphadenitis.

\section{Material and Methods}

Setting, Duration and type of study: Total of 50 cases, suspicious of tuberculous lymphadenitis presented with clinically palpable lymphnodes were included in study This study was conducted over 1.5 years period in 2018-19 at Pathology department of Government Medical College, Bhavnagar

Sampling methods: Patients were examined and written consent in local language was taken for the fine needle aspiration procedure.

Inclusion criteria: Clinically suspected cases of tuberculous lymphadenitis, clinically palpable lymphnodes.
Exclusion criteria: Inadequate aspirates or samples on which cytopathological diagnosis could not be given; Patients who refuse to give consent.

Data collection procedure: After adequate fine needle aspiration, aspirates were smeared onto three glass slides.

Out of them first was wet fixed smear stained with hematoxylin and eosin; second heat fixed smears were stained with Ziehl- Neelsen method and third for Auraminerhodamine stain.

Data analysis: sensitivity, specificity and accuracy calculated by using medcalc statistical software.

Ethical consideration \& permission: Institutional Review Board (Human Ethics Committee) of Government Medical College, Bhavnagar, Gujarat approval was obtained before study commencement.

The study was conducted accordance to Indian GCP guideline. The study has been registered at the Clinical Trial Registry of India.

Scoring system: Cytological findings, $\mathrm{ZN}$ stain observations and grading were compared with fluorescent stain grading [5].

\section{Result}

In present study maximum numbers of tuberculous lymphadenitis cases were observed in the age group of 21-30 years of age (15 cases, $30 \%$ ), followed by $11-20$ years (10 cases, $20 \%$ ) and $0-10$ years ( 9 cases, $18 \%$ ). Out of 50 male cases were 26 (52\%) and female $24(48 \%)$. most common site of tuberculous lymphadenopathy in the present study is cervical region (62\% cases), followed by submandibular (8\%), submental (8\%) and supraclavicular (8\%) regions.

Least common sites observed are inguinal and postauricular region with $2 \%$ cases of each.

Table-1: The grading of tuberculous lymphadenitis by fluorescent staining and ZN staining for acid fast bacilli for M. tuberculosis.

\begin{tabular}{|l|l|l|l|}
\hline Fluorescent Grading & No. of cases (\%) & ZN Stain Grading & No. of cases (\%) \\
\hline+3 & $14(28 \%)$ & +3 & $4(8 \%)$ \\
\hline+2 & $9(18 \%)$ & +2 & $14(28 \%)$ \\
\hline+1 & $11(22 \%)$ & +1 & $7(14 \%)$ \\
\hline Scanty & $2(4 \%)$ & Scanty & $4(8 \%)$ \\
\hline Negative & $14(28 \%)$ & Negative & $21(42 \%)$ \\
\hline Total & $\mathbf{5 0 ( 1 0 0 \% )}$ & Total & $\mathbf{5 0}(\mathbf{1 0 0} \%)$ \\
\hline
\end{tabular}

As shown in the Table 1, the maximum numbers of positive cases in fluorescent grading for M. tuberculosis were observed as grade +3 (14 cases, $28 \%$ ), followed by grade +1 (11 cases, $22 \%)$.

Maximum numbers of positive cases in RNTCP grading for AFB were observed as grade +2 (14 cases, $28 \%$ ), followed by grade +1 (7 cases, 14\%). Grading is fairly better with Fluorescent stain.

Pathology Update: Tropical Journal of Pathology \& Microbiology Available online at: www.medresearch.in 1016|P a g $e$ 
Table-2: Results of FNAC for the diagnosis of tuberculosis lymphadenitis by Ziehl- Neelsen and fluorescent methods for detecting tuberculous bacilli.

\begin{tabular}{|c|c|c|c|c|c|}
\hline \multirow[t]{2}{*}{ cytological diagnosis } & \multirow[t]{2}{*}{ Total Cases } & \multicolumn{2}{|c|}{ ZN staining } & \multicolumn{2}{|c|}{ Fluorescent staining } \\
\hline & & Positive & Negative & Positive & Negative \\
\hline Granulomatous lymphadenitis & 43 & 27 & 16 & 34 & 9 \\
\hline $\begin{array}{l}\text { Other than granulomatous } \\
\text { lymphadenitis }\end{array}$ & 7 & 2 & 5 & 2 & 5 \\
\hline Total & 50 & 29 & 21 & 36 & 14 \\
\hline
\end{tabular}

As shown in Table 2, cytological diagnosis as granulomatous lymphadenitis with AFB positivity on ZN staining was correlated in 27 cases. 2 cases were detected AFB positive on $\mathrm{ZN}$ stain but cytomorphologically not evident as granulomatous lymphadenitis. Whereas, cytological diagnosis as granulomatous lymphadenitis with positivity on fluorescent staining was correlated in 34 cases. 2 cases were detected positive on fluorescent stain but cytomorphologically not evident as granulomatous lymphadenitis.

Table-3: Sensitivity, specificity and accuracy of cytological diagnosis and ZN staining.

\begin{tabular}{|c|c|c|}
\hline Statistical measure & ZN staining & Fluorescent Microscopy \\
\hline Sensitivity & $93.10 \%$ & $94.44 \%$ \\
\hline Specificity & $23.81 \%$ & $35.71 \%$ \\
\hline accuracy & $64.00 \%$ & $78.00 \%$ \\
\hline
\end{tabular}

Sensitivity, Specificity and Accuracy of both the diagnostic tool are shown in table no 3 . Z $-\mathrm{N}$ stain is sensitive to detect tuberculous bacilli but specificity and accuracy of Ziehl-neelsen method is lower as compare to Fluorescent Microscopy.

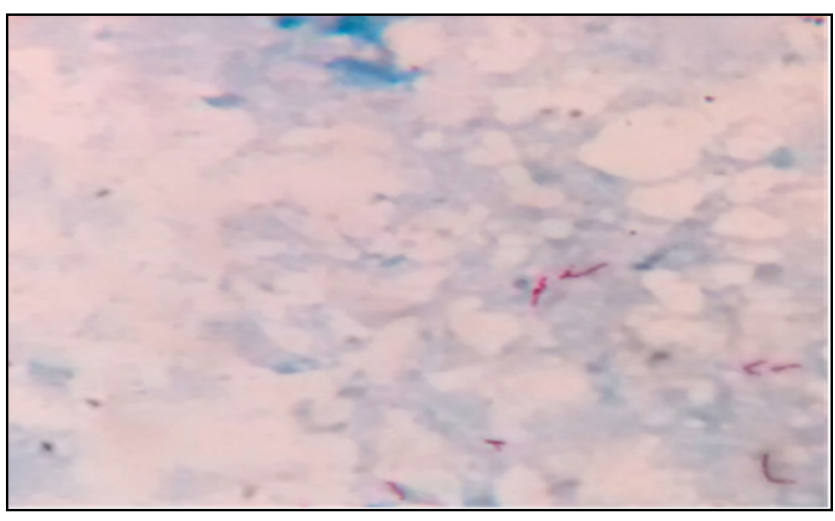

Fig-1: Ziehl-Neelsen stain showing acid fast tuberculosis bacilli.

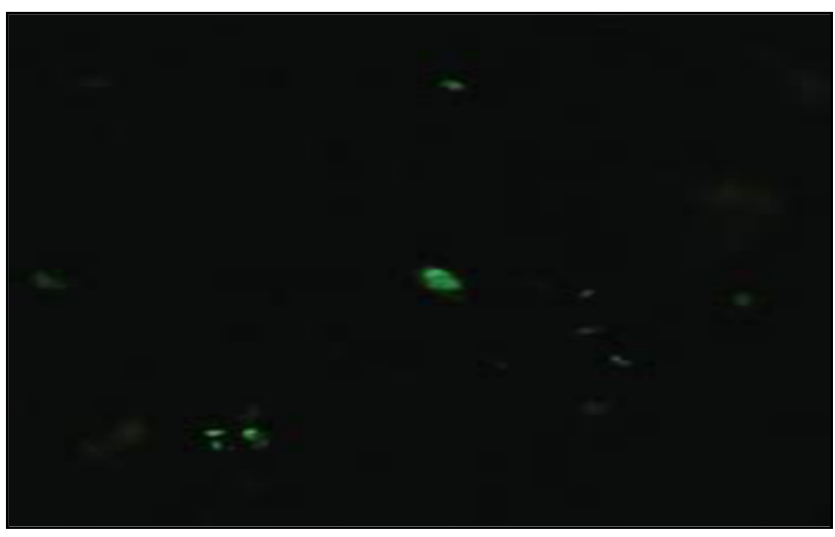

Fig-2: Fluorescent stain showing green rod shaped tuberculosis bacilli. 


\section{Discussion}

FNAC is an easy, reliable outpatient procedure for the diagnosis of tubercular lymphadenitis in palpable superficial lymph nodes, and it is ideally suited for use in resource limited settings, especially in developing countries where tuberculosis is a major cause of morbidity and mortality [6]. Conventional Ziehl-Neelsen (ZN) method for acid-fast bacilli (AFB) plays a key role in the diagnosis and the monitoring of treatment in tuberculosis.

Its major disadvantages are low sensitivity, time consuming, and oil immersion use. [4]. Fluorescent microscopy plays an important role for detection of Mycobacteria because lower magnifications are used as well as less time is required to examine smears. Fluorescence microscopy using auramine-rhodamine (AR) or Papanicolaou (PAP) staining has been considered to be superior to ZN staining [7]

Annam $\mathrm{V}$ et al [4] studied 102 lymph node aspirates, $44.11 \%(45 / 102)$ were positive for AFB on the conventional ZN method, 58.9\% (60/102) were indicative of TB on cytology, while the smear positive increased to $81.37 \%$ (83/102) on the modified fluorescent method. They concluded that the modified fluorescent method was found to be more advantageous than routine cytology and conventional $\mathrm{ZN}$ method, particularly in paucibacillary cases. The bacillary positivity rates were higher in the modified fluorescent method than in the ZN method.

Hence, the modified fluorescent method can be an adjuvant when used with routine cytology for the identification of AFB. In study of Brijesh Thakur et al [8] ninety aspirates were reported on cytomorphology as suggestive of tuberculous lymphadenitis. Smear positivity for Mycobacteria by Ziehl-Neelsen method was $26.67 \%$ (24/90), while positivity increased to $34.44 \%$ (31/90) by auramine-rhodamine and $42.22 \%(38 / 90)$ on autofluorescence. They Concluded that there is a definite advantage of autofluorescence over Ziehl-Neelsen and auramine-rhodamine which is to detect Mycobacteria, being more sensitive as well as an inexpensive technique.

Autofluorescence can be a useful addition to routine cytology for early diagnosis and effective treatment. Mani Krishna et al [9] studied among the clinically suspected patients 88 were diagnosed with tuberculosis. Of the 88 aspirates, the smear positivity for acid fast bacilli (AFB) on the $\mathrm{ZN}$ method was $37.5 \%(33 / 88)$ while the positivity increased to $81.82 \%(72 / 88)$ on the AR fluorescent method and $86.36 \%(76 / 88)$ on $\mathrm{AF} \mathrm{AF}$ staining is more sensitive than the auramine - rhodamine fluorescent and $\mathrm{ZN}$ staining in demonstration of mycobacterium bacilli in fine needle aspiration cytology of tubercular lymphnode.
Vamseedhar Annam et al [10] observed among the 102 aspirates, $44.11 \%$ (45/102) were positive for AFB on the conventional $\mathrm{ZN}$ method, $58.9 \%(60 / 102)$ were indicative of TB on cytology, while the smear positive increased to $81.37 \%$ (83/102) on the modified fluorescent method. Fluorescent microscopy has the advantage of speed and ease of screening and reduces observer fatigue.

In developing countries, microscopy of the specimen is by far the fastest, cheapest, and most reliable method for the detection of AFB. However fluorescent staining has been added in Revised National Tuberculosis Control Program (RNCP) because of more sensitive and rapid results and can be used in field areas. In a study done by Ben et al [11] in 2008 , showed that out of 221 sputum samples, 33(14.9\%) samples were positive with Auramine staining and 24 (10.85\%) samples were positive with Ziehl-Nelson staining. It demonstrated superior diagnostic results by fluorescent microscopy when compared with conventional light microscopy. Laifangbam et al [12] in 2009 revealed a study on 102 suspected patients, where in $44.1 \%$ patients were positive with Ziehl Nelson staining and $71.6 \%$ patients were positive for Auramine $\mathrm{O}$ staining. It was found by this study that Auramine O staining was superior to Ziehl Nelson staining. In the study by Vidhi sahani et al [13], they concluded that the Auramine $\mathrm{O}$ staining is superior to that of the conventionally used ZN stain. It states that out of 300 sputum samples; $32(10.66 \%)$ samples were $\mathrm{ZN}$ positive and 55(18.33\%) samples were Auramine O positive. The diagnostic accuracy of fluorescent microscope was found much more superior and much more sensitive than the conventional light microscopy.

In the present study, out of 50 cases, suspicious of tuberculous lymphadenitis presented with clinically palpable lymph nodes were underwent for FNAC procedure. Out of them 43 cases were diagnosed as granulomatous lymphadenitis. On examination by ZiehlNeelsen staining for acid fast bacilli 29 cases (58\%) were found positive. On fluorescent microscopy 34 cases (79.06\%) were positive. This result correlates well with the result of the other studies like Lokeshwaran et al [14] and Maru et al [15] observed $44 \%$ and $43.07 \%$ AFB positive cases on $\mathrm{ZN}$ stain. In the present study, it was observed higher sensitivity, specificity and accuracy with Fluorescent Microscopy. In the present study, the qualitative result of Fluorescent Microscopy was also found to be slightly better to the $\mathrm{ZN}$ stain, this is consistent with a study carried out by S. Laifangbam [12], which showed that the percentage of false negative by FM staining was only $2.78 \%$, and was in sharp contrast to that of $\mathrm{ZN}(40.27 \%)$. Fluorescent microscopy was also able to detect more paucibacillary cases than $\mathrm{ZN}$. 
The clinical parameters for the diagnosis of TB in lymph nodes are neither specific nor does their absence exclude TB involvement. Conventional Ziehl-Neelsen (ZN) method for AFB plays a key role in the diagnosis and monitoring of TB treatment. Its major disadvantage is its low sensitivity, ranging from $20 \%$ to $43 \%[16,17]$.

Fluorescent stain definitely has advantage over $\mathrm{ZN}$ stain probably because of an added advantage of fluorescent microscope which enables an easier detection of mycobacteria as 'glowing spot' even if, present in small numbers, thus making it a more sensitive technique. Despite some evidence that the fluorescent method can be superior to the $\mathrm{ZN}$ method, it is not being performed in peripheral tuberculosis laboratories in low-income countries. Newer methods using nucleic acid amplification techniques are gaining attention as a possible tool in diagnosing mycobacterial infections. These methods are rapid and potentially sensitive if sampling of organisms is adequate.

However, they are highly expensive [4]. Routine cytological staining method is fairly reliable with $96.77 \%$ sensitivity. If it is combined with $\mathrm{ZN}$ staining for AFB, specificity increases to $76.92 \%$ and even more if combined with Fluorescent Microscopy. Diagnosis of extrapulmonary tuberculosis is challenging due to the paucibacillary nature as well as atypical clinical presentations. Hence, its diagnosis should be made by considering more than one diagnostic method

\section{Conclusion}

There are problems in arriving at an absolute diagnosis in certain cases of tuberculous lymphadenitis when the aspirate shows polymorphous picture with occasional epithelioid cells and absence of typical Langhans giant cell or caseous necrosis. Microscopy has many advantages when it comes to speed and feasibility, its sensitivity could be improved by adjuvant methods, and it has the potential to become an even more valuable tool for TB control programs around the world.

Fluorescent method is extremely sensitive for detection of mycobacteria in the FNA smears. The sensitivity of conventional Fluorescent Microscopy provides far better yield and detection of positive smears than the $\mathrm{ZN}$ and takes less time to perform.

\section{What the study adds to the existing knowledge?}

This study explores the utility of fluorescent Microscopy as an adjunct to routine cytology as it is simple, rapid, and cost-effective screening technique especially in developing countries where tuberculosis is widely prevalent and shows continued presence of infection in the community.

\section{Author's contribution}

Dr. Alpeshpuri P. Goswami: Conception and design, Analysis and interpretation of the data, Drafting of the article, Final approval of the article, Administrative, technical, or logistic support, Critical revision of the article for important intellectual content

Dr. Smit Brahmbhatt: Provision of study materials or patients, Statistical expertise, Collection and assembly of data.

Dr. Abhijeet Khandkar: Analysis and interpretation of the data

Dr. Umesh Zinzala: Analysis and interpretation of the data Dr. Jignesh Gondliya: Provision of study materials or patients, Statistical expertise, Collection and assembly of data.

Dr. Parth Goswami: Critical revision of the article for important intellectual content, Collection and assembly of data.

Funding: No funding sources

Conflict of interest: None declared

Ethical Approval: This study was approved by the Institutional Ethics Committee

\section{References}

1. Raviglione MC, O'Brien RJ. Mycobacterial diseases: Tuberculosis. Harrison's Principles of Internal Medicine $19^{\text {th }}$ Edition. New York: McGraw Hill Education. 2015: 1102-1122.

2. TB India 2019 revised National TB control programme annual report 2019.

3. Daniel TM. Rapid diagnosis of tuberculosis: laboratory techniques applicable in developing countries. Rev Infect Dis. 1989;11(2):S471-S478.

4. Annam V, Kulkarni MH, Puranik RB. Comparison of the modified fluorescent method and conventional ZiehlNeelsen method in the detection of acidfast bacilli in lymphnode aspirates. Cyto J. 2009;6:13. doi: 10.4103 / 1742-6413.53887.

5. Reza LW, Satyanarayna S, Enarson DA, Kumar AM, Sagili K, Kumar S, et al. LED-fluorescence microscopy for diagnosis of pulmonary tuberculosis under programmatic conditions in India. PLoS One. 2013; 8(10):e75566. doi: https://doi.org/10.1371/journal.pone.0075566.

6. Wright CA, Hesseling AC, Bamford C, Burgess SM, Warren R, Marais BJ. Fine-needle aspiration biopsy: a firstline diagnostic procedure in paediatric tuberculosis suspects with peripheral lymphadenopathy? Int $\mathrm{J}$ Tubercul Lung Dis. 2009;13(11):1373-1379. 
7. A. Jain, A. Bhargava, and S. K. Agarwal, A comparative study of two commonly used staining techniques for acid fast bacilli in clinical specimens. Ind J Tuber. 2002;49:161162.

8. Brijesh Thakur, Ravi Mehrotra, Jitendra Singh Nigam Correlation of Various Techniques in Diagnosis of Tuberculous Lymphadenitis on Fine Needle Aspiration Cytology. Pathol Res Int.2013; 824620. doi: http://dx. doi. org/10.1155/2013/824620.

9. Krishna M, Kumar A, Tuberculous mycobacteria bacilli fluorescence and compare with Ziehl- Neelsen stain in fineneedle aspiration cytology of tubercular lymphnode. Int $\mathrm{J}$ Otorhinolaryngol Head Neck Surg. 2016;2(2):66-69. doi: http:// dx.doi.org/10. 18203/issn. 2454-5929. Ijohns 2016 0956.

10. Vamseedhar A, Kulkarni MH, Puranik BR. Comparison of the modified fluorescent method and conventional ZiehlNeelsen method in the detection of acidfast bacilli in lymphnode aspirates Cyto J. 2009;6(1):13. doi: 10.4103/ 1742-6413.53887.

11. Ben-Selma W, Ben-Kahla I, Marzouk M, Ferjeni A, Ghezal S, Ben-Said M, et al. Rapid detection of Mycobacterium tuberculosis in sputum by Patho-TB kit in comparison with direct microscopy and culture. Diagn Microbiol Infect Dis. 2009;65(3):232-235. doi: 10.1016/j. diagmicrobio.2009.07.021. Epub 2009 Sep 2.
12. Laifangbam S, Singh HL, Singh NB, Devi KM, Singh NT.A comparative study of fluorescent microscopy with Ziehl-Neelsen staining and culture for the diagnosis of pulmonary tuberculosis. Kathmandu Univ Med J (KUMJ). 2009; 7(27):226-230.

13. Sahani V, Karnal A, Arora S. A comparative study of ziehl-nelson staining and auramine staining in sputum sample for the diagnosis of pulmonary tuberculosis.2017; $6(6)$

14. Lokeshwaran, R.S., Lavanya, K.B., Dhanalakshmi, A., Keerthivasan, S. and Yogalakshmi, S., 2018. Comparison of auramine-o stain and ziehl-neelsen stain in the detection of tuberculous bacilli in fine needle aspiration of lymph nodes.JEvol Med Dent Sci-JEMDS. 2018;7(49):5268-5272

15. Maru AM, Kapadiya DN, makavana H, Lakum NR. Comparison of Z. N. staining \& fluorescent microscopy in detection of M. tuberculosis bacilli in Fine needle aspiration smears. Trop J Path Micro 2018; 4(3): 242-247.doi:10. 17511 / jopm. 2018. I3.02.

16. Daniel TM. Rapid diagnosis of tuberculosis: Laboratory techniques applicable in developing countries. Rev Infect Dis. 1989;11(2): S471-S478.

17. Balows A, Hausler WJ, Hermann KL, Shadomy HJ. Manual of clinical Microbiology. $5^{\text {th }}$ ed. Washington: D.C: American society for Microbiology; 1991. pp. 308311. doi: http://dx.doi.org/10.1590/S0036-46651991000 600014.

\section{How to cite this article?}

Goswami A.P, Brahmbhatt S, Khandkar A, Zinzala U, Gondliya J, Goswami P. Comparison of routine cytology findings with conventional Ziehl-Neelsen method and fluorescent method for detection of acid-fast bacilli in fine needle aspirates from suspected cases of tuberculous lymphadenitis. Trop J Path Micro 2019;5(12):1015-1020.doi:10.17511/ jopm.2019.i12.08 